\title{
Three-Phase Busbar Current Transducer
}

\author{
Andrey Chirtsov, Pavel Ripka, and Vaclav Grim \\ Faculty of Electrical Engineering, Czech Technical University, Prague, Czech Republic
}

\begin{abstract}
Receiv ed 1 Apr 2016, rev ised 15 Apr 2016, accepted 20 Apr 2016, published 1 Jun 2016, current version 15 Jun 2016. (Dates will be inserted by IEEE; "published" is the date the accepted preprint is posted on IEEE Xplore ${ }^{\circledR}$; "current version" is the date the typeset version is posted on Xplore ${ }^{\circledR}$.
\end{abstract}

\begin{abstract}
A true three-phase $1000 \mathrm{~A}$ busbar current transducer is based on 6 microfluxgate sensors. Instead of using three independent single-phase current transducers, we utilize the full information from each sensor. Two TI DRV425 microfluxgate sensors are inserted into the drilled hole of each busbar. Our method of data processing is optimized to compensate the crosstalk betw een three phases and also external fields and gradients up to $2^{\text {nd }}$ order. The achieved crosstalk compensation betw een the phases was $0.23 \%$ w orst case. By using the suggested method, the suppression of the external current in the distance of $10 \mathrm{~cm}$ is improved by the factor of 25 to 150 . Our transducer has the compact size, high temperature offset stability of $8.5 \mathrm{~mA} /{ }^{\circ} \mathrm{C}$, high current range up to $1000 \mathrm{~A}$, low pow er consumption and linearity of $0.1 \%$. The results from 3D-FEM model and analytical computations are confirmed by the measurements.
\end{abstract}

Index Terms — Electromagnetics, current sensor, busbar sensor, integrated fluxgate, microfluxgate.

\section{INTRODUCTION}

Busbars are used for distribution of high currents inside racks and also at electrical switchyards and stations. Modern grids and electric drives require to measure currents in individual busbars [Mota 2015, Salman 2017]. With the use of transformerless inverters, the requirement for the current sensors is wide bandwidth including the DC component, which is generated during transients and due to nonsymmetry in switching times and switched-on resistances for both polarities [Buticchi 2009].

Traditional busbar current transducers use Hall sensor inside the airgap of yoke surrounding the measured conductor. Yoke is effective in shielding external magnetic fields, but makes the transducer bulky, non-linear, temperature dependent and susceptible to remanence [Xiang 2018]. The common trend is therefore to develop yokeless current transducers using multiple magnetic sensors [Ripka 2019a].

The simplest solution is to put magnetic sensor on the surface of the busbar [Blagojevic 2016]. The disadvantage of this method is that the magnetic field in a near vicinity of the conduct or is high: for $60 \times 10$ mm busbar and current of $1000 \mathrm{~A}$ the field on the busbar surface is approximately $7 \mathrm{kA} / \mathrm{m}$. This limits the sensor selection to Hall sensors with their poor DC stability.

By using differential (gradiometric) configuration of sensors, one can make the transducer insensitive to external homogenous magnetic field, but not to field gradients [Zhang 2018, Blagojevic 2018]. In order to further increase the resistance against external currents, more magnetic sensors can be used either in circular [Itzke 2018, Yu 2018, K.L. Chen 2011; W.L. Chen 2018; T sai 2014] or rectangular array.

Another approach is to insert a couple of sensors into the hole drilled in each busbar. This solution allows to adjust the sensitivity and thus the range by changing the distance between the sensors [Snoeij 2016]. In this way transducers with $\mathrm{kA}$ range can be constructed using microfluxgate sensors with $2 \mathrm{mT}$ full scale range.

In this paper we extend the busbar current transducer described in
[Snoeij 2016] to measure three-phase currents. Instead of using three conventional single-phase current transducers, each with two magnetic sensors in differential mode, we developed true three-phase transducer by using more complex processing of signal from all six magnetic sensors. The target is to achieve no crosstalk between the phases and to minimize the influence of external magnetic fields, including those generated by other conductors. Similar approach was used for overhead current lines - in that case the conductor size can be neglected in comparison to the distance of the sensors; it is also easy to use higher number of sensors and distances of disturbing currents from overhead lines are high [Wu 2016, Bernieri 2017, Ripka $2019 \mathrm{~b}$ ]. In the present case of sensors inside the massive busbar, the calculation is more complicated, as we cannot neglect the conductor size. We can also expect disturbing currents in close busbars, which is typical for racks.

First, we explain the transducer design and derive analytical formulas for its description. 3-D FEM in ANSYS Maxwell is used for optimization. The results of calculations and modelling are finally verified by measurement.

\section{TRANSDUCER DESIGN}

The transducer geometry is shown in Fig. 1. Three 60x10 mm copper busbars are located in lateral plane in $160 \mathrm{~mm}$ distance which is standard in racks. Each busbar has circular hole with diameter of $\mathrm{d}$ $=19 \mathrm{~mm}$.

The pair of the microfluxgate sensors TI DRV425 with the fullscale range of $2 \mathrm{mT}$ is inserted into the hole in each copper busbar, therefore, our configuration utilizes in total six microflux gate sensors. For the distance between the sensors of $2 \mathrm{~s}=2.5 \mathrm{~mm}$, the sensitivity of the differential sensor pair to DC measured current calculated by FEM is $\alpha=1.6(\mathrm{~A} / \mathrm{m}) / \mathrm{A}$, this value was also verified experimentally. For $50 \mathrm{~A}$ DC current the field difference $\mathrm{H}_{\mathrm{FEM}}=81 \mathrm{~A} / \mathrm{m}, \mathrm{H}_{\text {meas }}=80$ $\mathrm{A} / \mathrm{m}$. The range of the current transducer for different applications can 
be easily optimized via busbar geometry, size of the hole and distance between two TI DRV425 sensors.

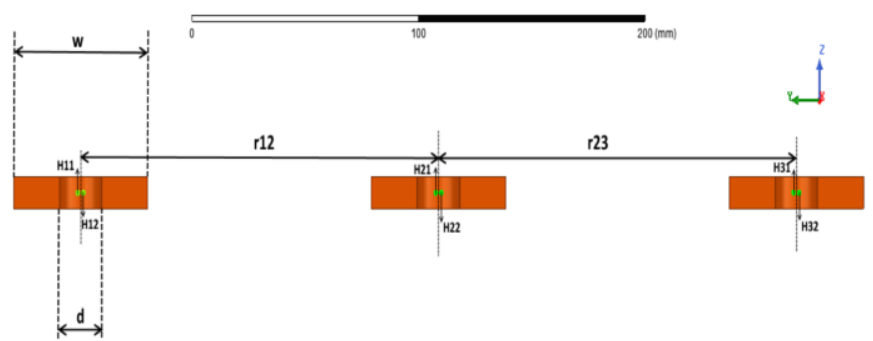

Fig. 1: Cross-section of the three-phase busbar system, green dots are the magnetic sensors

\section{THEORETICAL FRAMEWORK}

The theoretical framework for the analytical model is developed. The Ampere's law is used for analytical calculation of the parasitic response to the external current [Ripka 2017]. For the differential configuration with the spacing of $2 \mathrm{~s}$, the parasitic response to the idealized external current $\mathrm{I}$ in the distance $v$ in the same plane is

$$
H_{1}-H_{2}=\frac{I}{\pi(v+2 s) v}
$$

The systems of the equations (2) is used for 6 sensors and 3 phase currents to suppress the external current $\mathrm{I}_{\text {ext }}$. The busbars are simplified for long distances as one infinitely small point. Compensation of the crosstalk error between the phases is done with (2).

$$
\left(\begin{array}{l}
H_{1} \\
H_{2} \\
H_{3} \\
H_{4} \\
H_{5} \\
H_{6}
\end{array}\right)=\left(\begin{array}{cccc}
-\alpha & \frac{1}{2 \pi\left(r_{12}+s\right)} & \frac{1}{2 \pi\left(r_{13}+s\right)} & \frac{1}{2 \pi\left(r_{e x t}+r_{13}+s\right)} \\
\alpha & \frac{1}{2 \pi\left(r_{12}-s\right)} & \frac{1}{2 \pi\left(r_{13}-s\right)} & \frac{1}{2 \pi\left(r_{e x t}+r_{13}-s\right)} \\
-\frac{1}{2 \pi\left(r_{12}-s\right)} & -\alpha & \frac{1}{2 \pi\left(r_{23}+s\right)} & \frac{1}{2 \pi\left(r_{\text {ext }}+r_{23}+s\right)} \\
-\frac{1}{2 \pi\left(r_{12}+s\right)} & \alpha & \frac{1}{2 \pi\left(r_{23}-s\right)} & \frac{1}{2 \pi\left(r_{\text {ext }}+r_{23}-s\right)} \\
-\frac{1}{2 \pi\left(r_{13}-s\right)} & -\frac{1}{2 \pi\left(r_{12}-s\right)} & -\alpha & \frac{1}{2 \pi\left(r_{e x t}+s\right)} \\
-\frac{1}{2 \pi\left(r_{13}+s\right)} & -\frac{1}{2 \pi\left(r_{12}+s\right)} & \alpha & \frac{1}{2 \pi\left(r_{\text {ext }}-s\right)}
\end{array}\right)\left(\begin{array}{c}
I_{1} \\
I_{2} \\
I_{3} \\
I_{\text {ext }}
\end{array}\right)
$$

where $\alpha$ is the current sensitivity of each sensor which depends on its distance $\mathrm{s}$ from the center of the busbar, $\mathrm{r}_{12}$ and $\mathrm{r}_{23}$ are denoted in Fig. $1, r_{13}=r_{12}+r_{23}, r_{\text {ext }}$ is the distance to the external current, $I_{1}, I_{2}$, $\mathrm{I}_{3}$ are the amplitudes of the three-phase currents, $\mathrm{I}_{\text {ext }}$ is the amplitude of the external disturbing current and $\mathrm{H}_{1}, \ldots, \mathrm{H}_{6}$ are the measured values by the corresponding sensors. If the distance to the external current is known, the amplitude of the external current can be easily calculated. But in practice, this distance is unknown, then the system of the equations is nonlinear and should be solved numerically, which is not practical for industrial applications. The other problem is that the disturbances often come from multiple sources i.e. from several current conductors and also from ferromagnetic objects near current transducers. Therefore, we decided to change the compensation method to (3), which compensates the gradients of the external fields, where $\mathrm{H}_{\text {ext0 }}, \mathrm{H}_{\text {ext1 }}, \mathrm{H}_{\mathrm{ext} 2}$ are the field gradients of $0^{\text {th }}, 1^{\text {st }}$ and $2^{\text {nd }}$ order, respectively. The external field can be approximated as

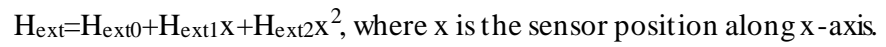

The Ampere's law which is used in our cases gives the result sclose to the 3D model in FEM only larger dist ances for which the conductor size is negligible. The exact analytical formula for the rectangular conductor was shown in [Olivares-Galvan 2009].

$$
\left(\begin{array}{l}
H_{1} \\
H_{2} \\
H_{3} \\
H_{4} \\
H_{5} \\
H_{6}
\end{array}\right)=\left(\begin{array}{cccccc}
-\alpha & \frac{1}{2 \pi\left(r_{12}+s\right)} & \frac{1}{2 \pi\left(r_{13}+s\right)} & 1 & 0 & 0 \\
\alpha & \frac{1}{2 \pi\left(r_{12}-s\right)} & \frac{1}{2 \pi\left(r_{13}-s\right)} & 1 & 2 s & (2 s)^{2} \\
-\frac{1}{2 \pi\left(r_{12}-s\right)} & -\alpha & \frac{1}{2 \pi\left(r_{23}+s\right)} & 1 & r_{12} & r_{12}^{2} \\
-\frac{1}{2 \pi\left(r_{12}+s\right)} & \alpha & \frac{1}{2 \pi\left(r_{23}-s\right)} & 1 & r_{12}+2 s & \left(r_{12}+2 s\right)^{2} \\
-\frac{1}{2 \pi\left(r_{13}-s\right)} & -\frac{1}{2 \pi\left(r_{12}-s\right)} & -\alpha & 1 & r_{13} & r_{13}^{2} \\
-\frac{1}{2 \pi\left(r_{13}+s\right)} & -\frac{1}{2 \pi\left(r_{12}+s\right)} & \alpha & 1 & r_{13}+2 s & \left(r_{13}+2 s\right)^{2}
\end{array}\right)\left(\begin{array}{c}
I_{1} \\
I_{2} \\
I_{3} \\
H_{\text {ext }} \\
H_{\text {ext }} \\
H_{\text {ext }}
\end{array}\right)
$$

The busbar of rectangular cross-section is considered with the length of the sides $2 a=60 \mathrm{~mm}$ and $2 b=10 \mathrm{~mm}$. Magnetic flux around the conductor can be calculated by (4).

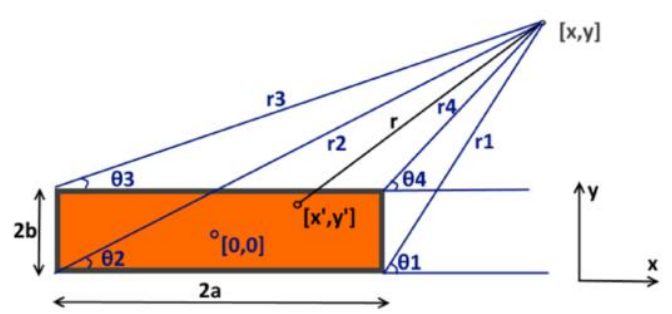

Fig. 2:2D theoretical schematic, cross-section of the busbar

$$
\begin{gathered}
A=\frac{I \mu_{0}}{16 \pi a b}\left\{(a-x)(b-y) \log \left((a-x)^{2}+(b-y)^{2}\right)+\right. \\
(a-x)(b+y) \log \left((a-x)^{2}+(b+y)^{2}\right)+ \\
(a+x)(b+y) \log \left((a+x)^{2}+(b+y)^{2}\right)+ \\
(a-x)^{2}\left(\arctan \frac{b-y}{a-x}+\arctan \frac{b+y}{a-x}\right)+ \\
(a+x)^{2}\left(\arctan \frac{b-y}{a+x}+\arctan \frac{b+y}{a+x}\right)+ \\
(b-y)^{2}\left(\arctan \frac{a-x}{b-y}+\arctan \frac{a+x}{b-y}\right)+ \\
\left.\quad(b+y)^{2}\left(\arctan \frac{a-x}{b+y}+\arctan \frac{a+x}{b+y}\right)\right\}
\end{gathered}
$$

$\mathrm{X}$ and $\mathrm{Y}$ component of the magnetic field strength can be calculated by (5) and (6) numerically or by using $\theta_{1}, \theta_{2}, \theta_{3}, \theta_{4}$ and $r_{1}, r_{2}, r_{3}, r_{4}$ in accordance with Fig. 2.

$$
\begin{aligned}
& H_{x}=\frac{1}{\mu_{0}} \frac{\partial A}{\partial y} \\
& H_{y}=-\frac{1}{\mu_{0}} \frac{\partial A}{\partial x}
\end{aligned}
$$

Then (5) and (6) converts to (7) and (8), respectively.

$$
\begin{gathered}
H_{x}=\frac{I}{8 \pi a b}\left\{-(y-b)\left(\arctan \frac{x-a}{y-b}-\arctan \frac{x+a}{y-b}\right)-\right. \\
(y+b)\left(\arctan \frac{x-a}{y+b}-\arctan \frac{x+a}{y+b}\right)+ \\
\left.(x+a) \log \frac{r_{3}}{r_{2}}-(x-a) \log \frac{r_{4}}{r_{1}}\right\} \\
H_{y}=\frac{I}{8 \pi a b}\left\{-(x-a)\left(\arctan \frac{y-b}{x-a}-\arctan \frac{y+b}{x-a}\right)-\right. \\
(x+a)\left(-\arctan \frac{y-b}{x+a}+\arctan \frac{y+b}{x+a}\right)+ \\
\left.(y+b) \log \frac{r_{1}}{r_{2}}-(y-b) \log \frac{r_{4}}{r_{3}}\right\}
\end{gathered}
$$

Where $r_{1}=\sqrt{(x-a)^{2}+(y+b)^{2}}, r_{2}=\sqrt{(x+a)^{2}+(y+b)^{2}}$, 


$$
r_{3}=\sqrt{(x+a)^{2}+(y-b)^{2}}, r_{4}=\sqrt{(x-a)^{2}+(y-b)^{2}}
$$

The equations (7) and (8) are the resulting equations which are used for individual busbar contributions. Fig. 3 shows that the calculated values fully correspond to the 2D FEM simulation. The largest difference is for $0.4 \mathrm{~mm}$ with corresponding error equal to $9.3 \%$, then the error is reduced to $3.7 \%$ at the distance of $10 \mathrm{~mm}$. The error for our configuration (for $160 \mathrm{~mm}$ distance between the centers of the busbar) equals to $0.02 \%$ and most likely it is caused by the inaccuracies in FEM simulations.

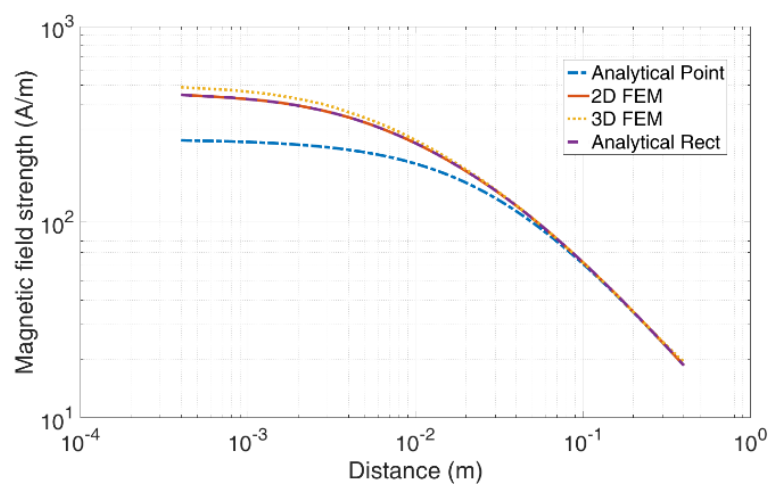

Fig. 3: Magnetic field strength in dependence on the distance from the busbar, comparison of all methods: Analytical calculation was made both neglecting sensor size (point) and considering real rectangular conductors (rect.)

\section{SIMULATION RESULTS}

3D model used for FEM simulations is shown in Fig. 5. For AC current the current distribution is no longer uniform due to the skin effect, and the sensitivity drops down with frequency. The distribution of the current density for $50 \mathrm{~Hz}$ and $1 \mathrm{kHz}$ is shown in Fig. 5 . Possibilities of frequency compensation were discussed in [Blagojevic 2018]. The conductivity of the busbar copper at room temperature was measured using four-terminal configuration and equals to $\sigma=5.55 \mathrm{MS} / \mathrm{m}$ and this value was used in simulations. The calculated comparison of the magnetic field strength inside the drilled hole for DC and AC f $=1 \mathrm{kHz}$ is shown in Fig. 6. Comparison between the new "compensated" method (suppression of the external fields and gradients) and "uncompensated" method (only crosstalk compensation) for lateral case (disturbing current in-plane with busbar system) is shown in Fig. 7. The reading error for $100 \mathrm{~mm}$ distance is reduced from $32 \%$ to $0.4 \%$ for $I_{1}$, which represents 80 fold error reduction. Error reductions for $\mathrm{I}_{2}$ and $\mathrm{I}_{3}$ were 150 and 25 respectively.

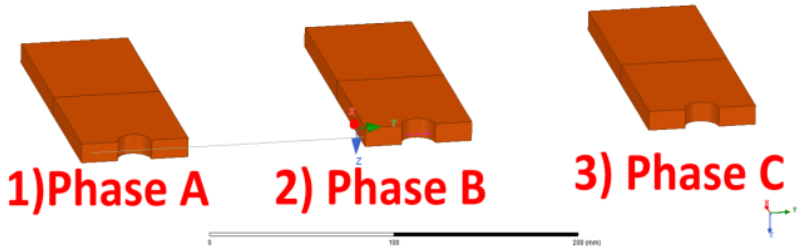

Fig. 4:3D FEM model in Ansys Maxwell

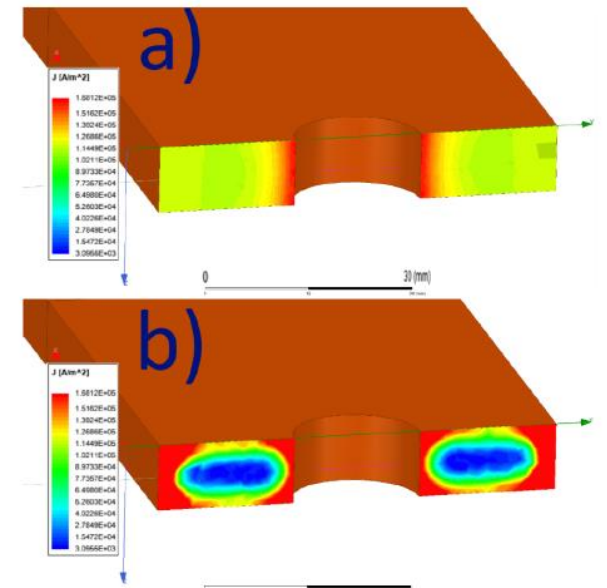

Fig. 5: Current distribution $I_{\max }=50 \mathrm{~A}$ a) $50 \mathrm{~Hz}$ b) $1 \mathrm{kHz}$

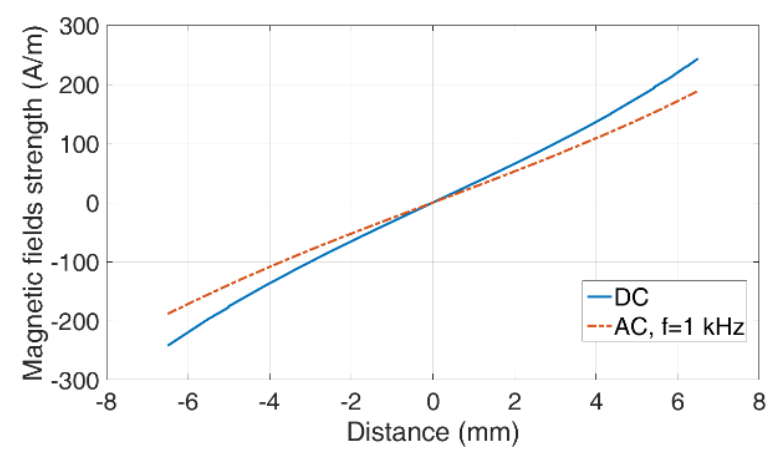

Fig. 6: Magnetic field strength inside the drilled hole for $50 \mathrm{~A}$ current, $0 \mathrm{~mm}$ corresponds to the center of the busbar

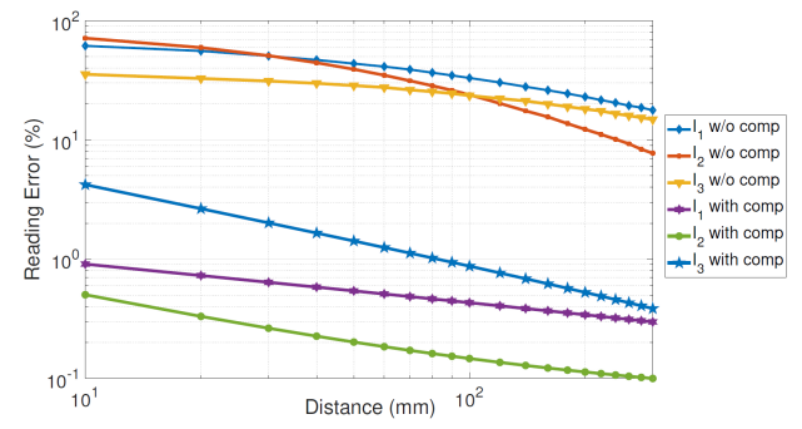

Fig. 7: External current error for each phase with compensation and without as a function of the distance of the lateral disturbing current (measured from the edge of $L_{3}$ as shown in Fig. 10). Calculated from FEM simulations for real size of the busbars.

\section{MEASUREMENTRESULTS}

One of the three busbars with the inserted sensor is shown in Fig. 9. The microfluxgate sensors are placed on both sides of PCB.

The star (wye) configuration is used for the test setup shown in Fig. 8 , where $\mathrm{B}_{1}, \mathrm{~B}_{2}, \mathrm{~B}_{3}$ are the copper busbars; $\mathrm{R}_{1}, \mathrm{R}_{2}, \mathrm{R}_{3}$ are the rheostats (6.3 A, 13 $\Omega$ ) which are used to control the flowing current and $\mathrm{R}_{4}$, $\mathrm{R}_{5}, \mathrm{R}_{6}$ are the reference resistors $0.01 \Omega$ which are used as the shunt resistors for precision current reading. The three-phase transformer $220 \mathrm{~V} / 24 \mathrm{~V}$ is used for feeding the busbars with the 3 phase $\mathrm{L}_{1}, \mathrm{~L}_{2}, \mathrm{~L}_{3}$ and the external conductor is in-phase with $\mathrm{L}_{2}$. The output signals from the 6 microfluxgate transducers are processed by 
multifunctional DAQ-Card NI-USB 6211 (16 bit, $250 \mathrm{kS} / \mathrm{s})$ and later by the LabVIEW program. The external current was placed in plane with three busbars and distance between the external conductor and our system was changed as shown in Fig. 10. The measurement results are shown together with the simulation results in Fig. 11. The maximum difference bet ween the measurement and simulation is $3 \%$.

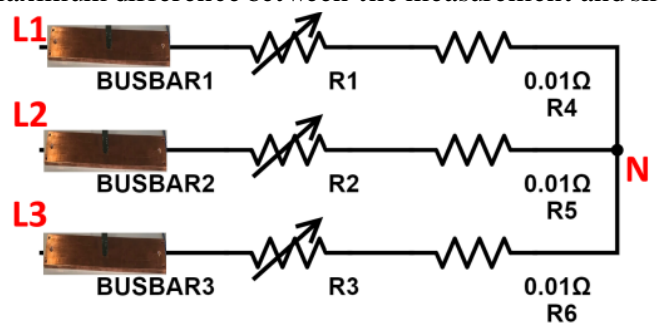

Fig. 8: Electrical connection of the three-phase system $L_{1}, L_{2}, L_{3}$ are the phases, $N$ - neutral line

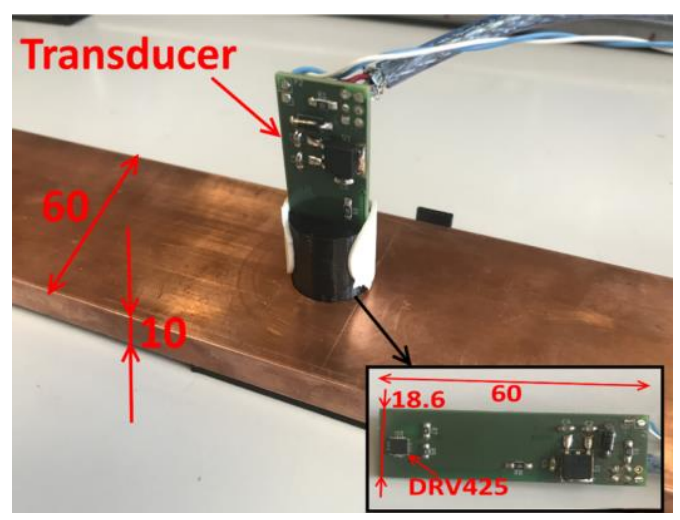

Fig. 9: One of the three busbar with the transducer in the working position

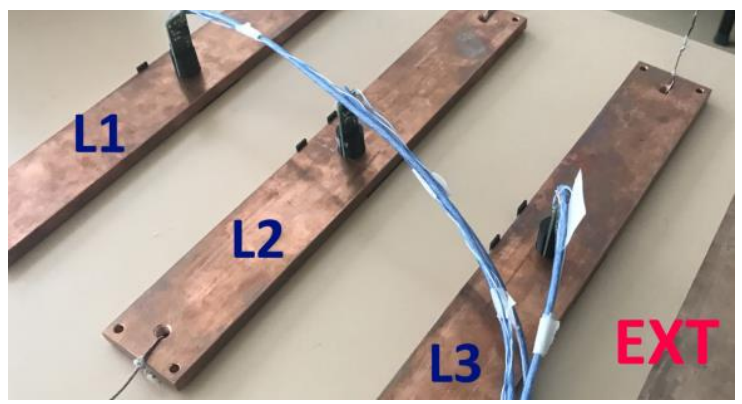

Fig. 10: Three-phase busbar system with the inserted sensors. L1, $\mathrm{L} 2$, $\mathrm{L} 3$ are the three phasesand EXT is the external conductor.

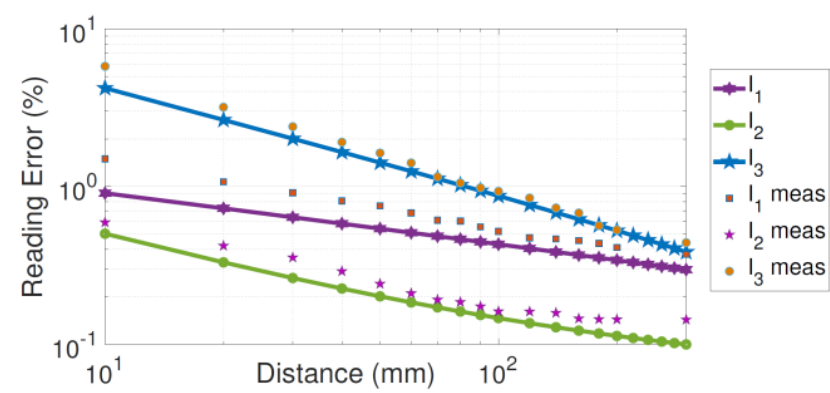

Fig. 11: External current error: comparison of the simulation results and measurements in dependence on the position of the in-plane external current

Current 5 A through each phase was subsequently applied to measure both the crosstalk error between the phases and difference between the measured and theoretically computed matrix (2). The results are shown in Table 1 and 2, respectively. Theoretically the crosstalk is zero, meaning that the currents in other phases does not influence the measured current. The main source for deviations are the geometrical inaccuracies and error of the sensor sensitivities. Table 1 is symmetrical, with max. $0.2 \%$ error. The largest errors are for the sensitivities $\alpha$ (bold in Table 2). These indicate the systematic $+1.6 \%$ error in estimating the sensor distance of $2.5 \mathrm{~mm}$.

Table 1: Crosstalkerror between the phases

\begin{tabular}{|c|c|c|c|}
\hline $\mathrm{I}_{1}-\mathrm{I}_{2}-\mathrm{I}_{3}(\mathrm{~A})$ & Error L1 (\%) & Error L2 (\%) & Error L3 (\%) \\
\hline $5-0-0$ & $\mathrm{X}$ & 0.2 & 0.07 \\
\hline $0-5-0$ & 0.23 & $\mathrm{X}$ & 0.19 \\
\hline $0-0-5$ & 0.09 & 0.18 & $\mathrm{X}$ \\
\hline
\end{tabular}

Table 2: Measured error (\%) for components of left sub-matrix (2)

\begin{tabular}{|c|c|c|}
\hline $\mathbf{1 . 6 0}$ & -0.34 & -0.11 \\
\hline $\mathbf{1 . 4 6}$ & 0.39 & 0.09 \\
\hline-0.28 & $\mathbf{1 . 5 7}$ & -0.4 \\
\hline 0.4 & $\mathbf{1 . 6 7}$ & 0.43 \\
\hline-0.13 & -0.24 & $\mathbf{1 . 7 9}$ \\
\hline-0.08 & 0.37 & $\mathbf{1 . 5 0}$ \\
\hline
\end{tabular}

\section{CONCLUSION}

The novel method for the measurement of the currents in threephase systems is presented in this paper. Our method theoretically completely suppresses crosstalk between the phases for arbitrary phase currents. The real crosstalk is $0.2 \%$. The external disturbances cannot be canceled completely - our new method suppresses the homogeneous component and external field gradients up to $2^{\text {nd }}$ order for 6 sensor configuration. With the increasing number of the operating sensor, the higher order of the field gradients could be suppressed, e.g. up to $4^{\text {th }}$ order for 8 sensors, and up to $6^{\text {th }}$ order for 10 sensors. Better suppression can be also achieved by using priory knowledge of the phase current such as their sum is zero. The resolution of tens $\mathrm{mA}$ is achievable since the microfluxgate sensors TI DRV425 have the small noise $(6 \mathrm{~mA} / \sqrt{\mathrm{Hz}}) @ 1 \mathrm{~Hz}$ with the fullscale range of $1000 \mathrm{~A}$ and low offset drift with the temperature 5 $\mathrm{nT} /{ }^{\circ} \mathrm{C}$, which corresponds to $8.5 \mathrm{~mA} /{ }^{\circ} \mathrm{C}$ for our solution. This is significantly better offset stability compared with AMR $\left(20 \mathrm{nT} /{ }^{\circ} \mathrm{C}\right)$ and the Hall sensor $\left(5 \mu \mathrm{T} /{ }^{\circ} \mathrm{C}\right)$. The power consumption is low $(<1 \mathrm{~W}$ for three transducers even for the maximum measured current of 1000 A); our transducer has linearity of $0.1 \%$, compact size and low price. The FEM results are confirmed by the measurements. The main disadvantage of this solution is the frequency dependence caused by eddy currents in the solid busbar.

\section{ACKNOWLEDGMENT}

The authors would like to thank Ing. Mehran Mirzaei for his advice and assistance in the analytical framework.

This work was supported by the Grant agency of the Czech Republic through the project "New methods for the measurement of electric currents" (GACR 17-19877S) and by Student Grant Agency ofCzech Technical University through the project "Novel magnetic sensors" (SGS18/187/OHK13/3T/13). 


\section{REFERENCES}

Bernieri, A., L. Ferrigno, M. Laracca, and A. Rasile. 2017. "An AMR-Based Three-Phase Current Sensor for Smart Grid Applications." Ieee Sensors Journal 17 (23): $7704-$ 7712. https://doi.org/10.1109/jsen.2017.2725983.

Blagojevic M, Jovanovic U, Jovanovic I, Mancic D, Popovic R S (2016) “Realization and optimization of bus bar current transducers based on Hall effect sensors," Measurement Science and Technology, Vol. 27: 065102.

Blagojevic M, Jovanovic U, Jovanovic I, Mancic D (2018) "Folded bus bar current transducerbased on Hall effect sensor." Electrical Engineering 100 (2): 1243-1251. https://doi.org/10.1007/s00202-017-0579-2

Buticchi, G., G. Franceschini, E. Lorenzani, C. Tassoni, A. Bellini, and Ieee. 2009. A Novel Current Sensing DCOffset Compensation Strategy in Transformer lessGrid Connected Power Converters.2009 Ieee Energy Conversion Congress and Exposition, Vols 1-6. New York: Ieee.

Chen, K. L., and N. M. Chen. 2011. "A New Method for Power Current Measurement Using a Coreless Hall Effect Current Transformer." Ieee Transactions on Instrumentation and Measurement 60 (1): 158-169. https://doi.org/10.1109/tim.2010.2049234.

Chen, W. L., H. Q. Zhang, L. Chen, and S. Y. Gu. 2018. "Wire-positioning algorithm for coreless Hall array sensors in current measurement." Measurement Science and Technology 29 (5): 5. https://doi.org/10.1088/1361-6501/aaaef0.

Itzke, A, Weiss R, T. DiLeo T, Weigel R (2018) The Influence of Interference Sources on a Magnetic Field-Based Current Sensor for Multiconductor Measurement. IEEEe Sensors Joumal, 18, 6782-6787.

Mota, L. T. M., A. D. Mota, and L. C. Coiado. 2015. "Non-Destructive Current Sensing for Energy Efficiency Monitoring in Buildings with Environmental Certification." Sensors 15 (7): 16740-16762. https://doi.org/10.3390/s150716740.

Ripka P, Chirtsov A (2017), "Influence of External Current on Yokeless Electric Current Transducers.” IEEE Trans. Magn. Vol. 53 (11) paper \# 4003904, 1-4 doi: 10.1109/TMAG.2017.2715075

Ripka P. (2019a), "Contactless measurement of electric current using magnetic sen sors", Technisches Messen, Early Access 10.1515/teme-2019-0032

Ripka P, Grim V, Chirtsov A (2019b) “A 3-Phase Current Transducer based on microfluxgate sensors," Measurement Vol. 146, November 2019, pp. 133-138 https://doi.org/10.1016/j.measurement.2019.06.028

Snoeij M F, Schaffer V, Udayashankar S, Ivanov M V (2016) Integrated Fluxgate Magnetometer for Use in Isolated Current Sensing. IEEE Journal of Solid-State Circuits, 51, 1684-1694.

Olivares-Galvan J C, Hernandez I, Georgilakis P S, Campero-Littlewood E (2009), "Calculation of the Magnetic Field Intensity in a Rectangular Conductor Canying Current in Electromagnetism Introductory Courses", Excerpt from the Proceedings of the COMSOL Conference 2009 Boston

Tsai, Y. P., K. L. Chen, Y. R. Chen, and N. M. Chen. 2014. "Multifunctional Coreless Hall-Effect Current Transformer for the Protection and Measurement of Power Systems." Ieee Transactions on Instrumentation and Measurement 63 (3): 557-565. https://doi.org/10.1109/tim.2013.2281555.

Wu, J. Y., Z. F. Chen, C. Wang, and L. L. Hao. 2016. "A Novel Low-Cost MulticoilBased Smart Current Sensor for Three-Phase Currents Sensing of Overhead Conductors." Ieee Transactions on Power Delivery 31 (6): 2443-2452. https://doi.org/10.1109/tpwrd.2015.2402284.

Xiang, Y. K., Q. F. Xu, Y. F. Huang, and H. Chen. 2018. "A Ferromagnetic Design for Current Sensor Temperature Characteristics Improvement." Ieee Sensors Joumal 18 (4): 1435-1441. https://doi.org/10.1109/jsen.2017.2785961.

Yu H, Qian Z, Liu H Y, Qu J Q (2018) "Circular Array of Magnetic Sens ors for Current Measurement: Analysis for Error Caused by Position of Conductor," Sensors 18 (2): 12. https://doi.org/10.3390/s18020578

Zhang, M. J., and S. W. Or. 2018. "Gradient-Type Magnetoelectric Current Sensor with Strong Multisource Noise Suppression." Sensors 18 (2): 16. https://doi.org/10.3390/s18020588. 